\title{
Early anisotropy changes in the corpus callosum of patients with optic neuritis
}

\author{
M. Bester • C. Heesen - S. Schippling • R. Martin • \\ X.-Q. Ding • B. Holst • J. Fiehler
}

Published online: 11 September 2008

(C) Springer-Verlag 2008

Erratum to: Neuroradiology (2008) 50:549-557

DOI 10.1007/s00234-008-0377-7

An erroneous number for the mean FA of the GCC in the control group was reported at MRI3 in the section "Follow up of white matter leasions, ADC and FA". It should read (0.72 vs $0.76, \mathrm{P}<0.01)$. Due to an oversight wrong $\mathrm{ADC}$ and FA values for the control group were reported in Table 2. The table should read as follows:

The online version of the original article can be found at http://dx.doi. org/10.1007/s00234-008-0377-7.

M. Bester $\cdot$ C. Heesen $\cdot$ S. Schippling $\cdot$ R. Martin $\cdot$ X.-Q. Ding $\cdot$

B. Holst $\cdot$ J. Fiehler $(\triangle)$

Department of Neuroradiology,

University Medical Center Hamburg-Eppendorf,

Martinistrasse 52,

20246 Hamburg, Germany

e-mail: fiehler@uke.uni-hamburg.de

C. Heesen $\cdot$ S. Schippling $\cdot$ R. Martin Institute for Neuroimmunology and Clinical MS Research,

University Medical Center Hamburg-Eppendorf,

Hamburg, Germany 
Table 2 ADC $\left(10^{-3} \mathrm{~mm}^{2} / \mathrm{s}\right)$ and FA values of patients and controls at nany specified time

\begin{tabular}{|c|c|c|c|c|c|c|c|c|c|c|c|c|c|c|c|}
\hline & \multicolumn{15}{|l|}{ MRI } \\
\hline & \multicolumn{3}{|l|}{1} & \multicolumn{3}{|l|}{2} & \multicolumn{3}{|l|}{3} & \multicolumn{3}{|l|}{4} & \multicolumn{3}{|c|}{ Control } \\
\hline & Mean & $\mathrm{n}$ & SD & Mean & $\mathrm{n}$ & SD & Mean & $\mathrm{n}$ & SD & Mean & $\mathrm{n}$ & SD & Mean & $\mathrm{n}$ & $\mathrm{SD}$ \\
\hline FWM ADC & 77.61 & 24 & 9.03 & 75.91 & 24 & 9.60 & 78.91 & 15 & 7.39 & 82.66 & 5 & 8.00 & 74.27 & 15 & 4.28 \\
\hline Genu ADC & 79.88 & 24 & 7.52 & 81.64 & 24 & 10.24 & 81.15 & 15 & 7.18 & 85.52 & 5 & 8.09 & 81.78 & 15 & 7.83 \\
\hline OWM ADC & 85.60 & 24 & 11.44 & 85.85 & 24 & 10.71 & 84.37 & 15 & 8.66 & 92.03 & 5 & 7.24 & 82.13 & 15 & 4.52 \\
\hline Splenium ADC & 78.55 & 24 & 7.30 & 77.50 & 24 & 5.51 & 81.60 & 15 & 8.60 & 79.99 & 5 & 5.94 & 77.54 & 15 & 7.03 \\
\hline FWM FA & 0.448 & 24 & 0.11 & 0.390 & 24 & 0.13 & 0.436 & 15 & 0.07 & 0.394 & 5 & 0.12 & 0.401 & 15 & 0.06 \\
\hline Genu FA & 0.725 & 24 & 0.09 & 0.714 & 24 & 0.10 & 0.721 & 15 & 0.08 & 0.619 & 5 & 0.07 & 0.764 & 15 & 0.06 \\
\hline OWM FA & 0.520 & 24 & 0.16 & 0.527 & 24 & 0.15 & 0.502 & 15 & 0.16 & 0.463 & 5 & 0.14 & 0.566 & 15 & 0.07 \\
\hline Splenium FA & 0.726 & 24 & 0.08 & 0.691 & 24 & 0.12 & 0.715 & 15 & 0.09 & 0.629 & 5 & 0.08 & 0.816 & 15 & 0.03 \\
\hline
\end{tabular}

